\title{
Ultra-Short Pulsed Laser Marking and Coloration of Metals with Segmented Pixel Parameter Transformation
}

\author{
Norbert Ackerl ${ }^{1}$, Pascal Gugger ${ }^{1}$, Maximilian Warhanek ${ }^{1}$, Johannes Gysel ${ }^{2}$ and Konrad Wegener ${ }^{1}$ \\ ${ }^{1}$ ETH Zurich, Institute of Machine Tools and Manufacturing (IWF), \\ Leonhardstrasse 21, 8092 Zurich, Switzerland. \\ E-mail: ackerln@ethz.ch \\ ${ }^{2}$ inspire AG, Technoparkstrasse 1, 8005 Zurich, Switzerland.
}

\begin{abstract}
Ultra-short pulsed laser modification points to novel coloration possibilities on a broad range of materials using laser induced periodic surface structures, plasmonic nanoparticles, and thermal oxidation as principles. Here, a general parameter-dependent laser path calculation routine for marking and coloration of complex images is presented. The original image is imported, dependent on size and resolution, down sampled and dithering applied. The color map attained from a laser parameter study is registered and the computer-aided manufacturing routine maps the color space to nearest neighbors of the original image. Thereafter, the laser paths with the adjacent parameters are computed and the numerical code generated. A complex colored pixel graphic is transformed and high-contrast marked in black and grey-scale on a stainless steel plate. Moreover, to demonstrate the capabilities, a titanium alloy is ultra-short pulsed laser colored. A colorful image proves this approach with a pixel size of below $40 \mu \mathrm{m}$ and no observable cross talk between the colors.
\end{abstract}

DOI: $10.2961 /$ jlmn.2020.01.2001

Keywords: laser marking, coloration strategies, ultra-short pulses, computer-aided manufacturing, pixel-to-path computation, tempering colors, laser induced periodic surface structures

\section{Introduction}

High-contrast laser marking is heavily used in industry to generate unique labels on workpieces and goods. Depending on the material to be tagged, adjacent absorptivity, and desired quality, different laser sources are utilized. Especially, the pulse duration gives rise to different physical mechanisms, which lead to contrast on the material. In principle, several mechanisms lead to a contrast on the material, which are i) removing of parts of the surface, ii) ablate a thin layer to reveal the e.g. anodized substrate iii) introducing absorbing surface structures, iv) tempering colors, v) generating diffraction gratings and plasmonic nanoparticles, and vi) introducing phase changes leading to contrast. The first three mechanisms point to binary or greyscale marking and are industrially implemented. Tempering colors are observed at different metallic compounds, where a thermally driven oxidation occurs [1,2]. However, these processes are challenging to control and often have a long processing time. Recently, ultra-short pulsed systems are available with good stability and reasonable price. This leads to novel marking and coloration possibilities on a broad range of materials utilizing e.g. laser induced periodic surface structures (LIPSS) as diffraction gratings [3-6], plasmonic nanoparticles [7], micro/nano surface roughness [8-10], and thermal oxidation for tempering colors. Recently, the laser marking of unique identification codes on medical devices with high corrosion resistance opened the new field of industrially ultra-short pulsed high-contrast marking [11].

Depending on the application different requirements appear. In rough environments, the corrosion resistance is an issue [12-14], whereas for aesthetic applications like jewelry the variety in colors and saturation is of more interest $[15,16]$. For both applications, high contrast and vivid colors are desired. Novel applications ask for the combination of marking and changing the surface properties, where for example the wettability, tribological, and optical properties can be changed simultaneously [17]. Potentially, ultra-short pulsed marking with LIPSS can be used to generate unique difficult to counterfeit structures in a fast manner [5,18]. Moreover, holographs from superposition of structures in the sub-micrometer regime are difficult to copy and the initial cost of ultra-short systems keep decreasing.

This contribution deals with the general parameterdependent laser path calculation for marking and coloration of complex pixelated images. A computer-aided manufacturing (CAM) tool is presented to directly determine the machine code for laser ablation on varying axis configurations. The necessary operations and used algorithms are discussed to reach marking in a fast forward way. To demonstrate the capabilities, a titanium alloy (TiAl6V4) is ultra-short pulsed colored with an Amphos 200 laser source with $1030 \mathrm{~nm}$ wavelength. Moreover, highcontrast marking of stainless steel with $515 \mathrm{~nm}$ wavelength is presented.

\section{Experimental Routines}

The laser marking and coloration can be carried out on different experimental testbeds. Depending on the specimen and size, a combination of mechanical axes and optical axes is necessary. Here, the pixel parameter transformation is in principle independent of the specific laser source. Nevertheless, LIPSS and nanoparticles demand an ultrashort pulsed laser source. In case of tempering colors, where the total introduced energy below the ablation threshold 
points to the oxide layer or formation of mixed valence, a nanosecond or continuous wave source fits better. A material-dependent parameter study is necessary to distinguish the adjacent setting for the laser modification process. A study shows coloration on a stainless steel, a titanium alloy (TiAl6V4), and an oxygen-free copper sample.

\subsection{Axes configuration and controllers}

The experimental setup used in this study is depicted in figure 1. An ultra-short pulsed Amphos 200 system is used as source and the laser beam is guided through optional wave plates altering the polarization state to the optical scanhead.

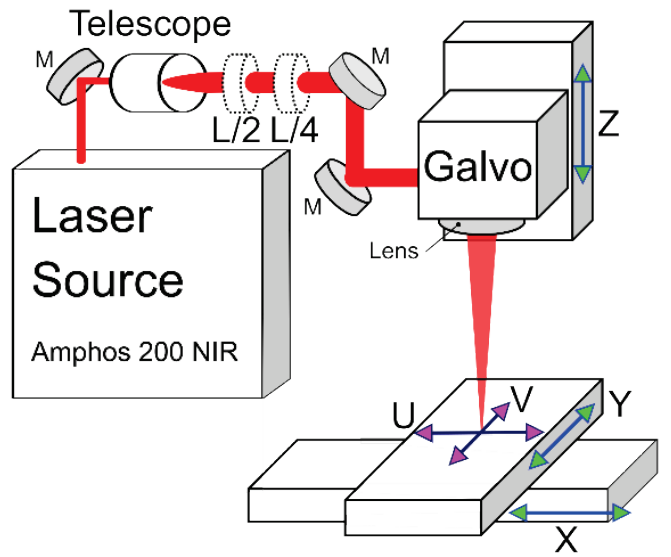

Fig. 1 Experimental setup with the laser source, open beam path with telescope and polarizing wave plates coupled into a galvo scan head with UV axes mounted on a focus Z-axis. Mechanical XY-axes allow positioning and stitching.

Furthermore, a telescope allows modifying the raw beam diameter and therefore focal radii. The scanhead is mounted on a mechanical axes system constituted by XYZ linear stages from Aerotech $\mathrm{GmbH}$. Controllers from the same company allow a flexible programming of laser scanning paths with a fast laser trigger.

\subsection{Tempering Colors on stainless stee}

Stainless steel is known to show colors after exposing it to elevated temperature in ambient condition for some time [19]. An iron oxide layer and underlying spinel structure chromium oxide leads to different colors. A parameter study on an austenitic $\mathrm{Cr} / \mathrm{Ni}$ stainless steel (1.4301) with fixed
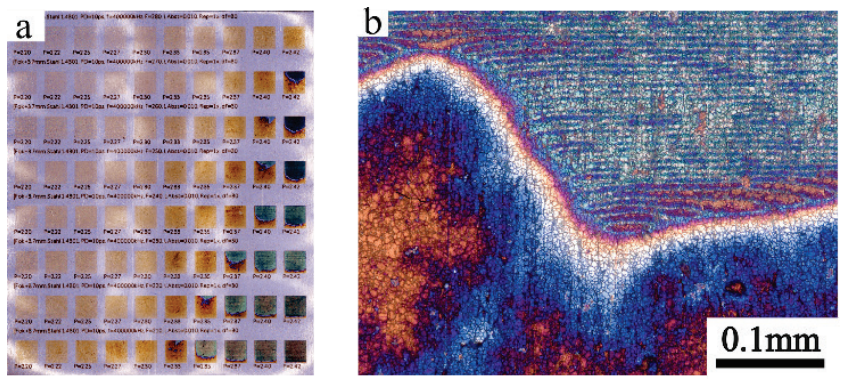

Fig. 2 Parameter study on 1.4301 stainless steel (a) varying scanspeed and power. (b) Reveals the color appearance at the point of plasma ignition.

$1030 \mathrm{~nm}$ wavelength, $10 \mathrm{ps}$ pulse duration, a repetition rate of $40 \mathrm{MHz}$, and laser line spacing of $10 \mu \mathrm{m}$ was carried out. The total energy input was controlled by the scan speed and the average power. Due to the low threshold fluence of metals, the beam was $3.7 \mathrm{~mm}$ defocused. Figure 2a shows the attained colors, where the lines depict the variation of scanspeed from $170 \mathrm{~mm} / \mathrm{s}$ to $280 \mathrm{~mm} / \mathrm{s}$ and the columns of average power between $12 \mathrm{~W}$ to $16 \mathrm{~W}$. Clearly, oxidation occurred, and the total energy input influences the color. The fields of $9 \mathrm{~mm}^{2}$ were scanned one time and after plasma ignition vivid colors occur. Interestingly, close to the onset of plasma a transition is observed. Figure $2 b$ shows this scenario happening e.g. at $15.4 \mathrm{~W}$ and a scan speed of $230 \mathrm{~mm} / \mathrm{s}$. Following, a defined coloration of stainless steel is challenging and the parameter space small.

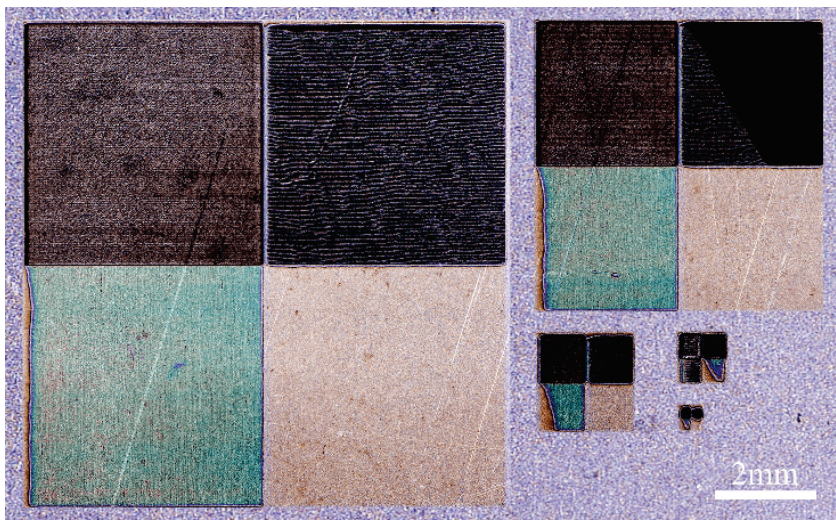

Fig. 3 Size dependent colors on stainless steel keeping the energy per unit area constant. The green color depends on marking size.

A study with multiple repetitions to introduce the same total energy revealed inconsistent coloration. Moreover, changing the size of a colored field influences the repeatability, shown in figure 3 . Already a small change in the heat accumulation makes a fast forward coloration subtle. However, the introduction of tempering colors bases on heating and, therefore, ultra-short pulses generally not necessary. A study in literature on the process robustness using $100 \mathrm{~ns}$ pulses reveals a rich color map in conjunction with stable size-independent processing [1].

\subsection{Tempering colors on titanium}

A study on a TiAl6V4 alloy covering a small range of parameters shows multiple stable colors in figure $4 \mathrm{a}$. The focal spot radii of the NIR beam was $35 \mu \mathrm{m}$ and the scan speed altered from $20 \mathrm{~mm} / \mathrm{s}$ up to $280 \mathrm{~mm} / \mathrm{s}$ with average powers between $1 \mathrm{~W}$ and $7 \mathrm{~W}$. Titanium has multi-valency
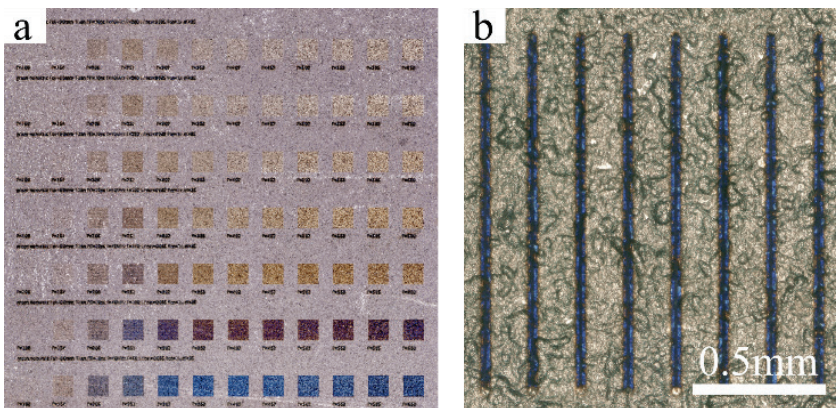

Fig. 4 Study on the coloration of TiAl6V4 in (a) and single line generated blue color in (b).

and forms five stable oxide configurations leading to a variety of colors [5]. Hence, the colors are very stable and a variation of field size gives persisting colors. Even the single lines in figure $4 \mathrm{~b}$ show a stable blue at small scale. This 
enables single focal diameter pixel sizes with little crosstalk and distinct color separation.

\subsection{LIPSS generation on aluminum and copper}

Laser-induced periodic surface structures have been studied extensively in literature in recent years [20]. These self-organized structures appear after multi ultra-short pulse irradiation of any material near the threshold fluence with linear polarized light. Here, a brief study shows the
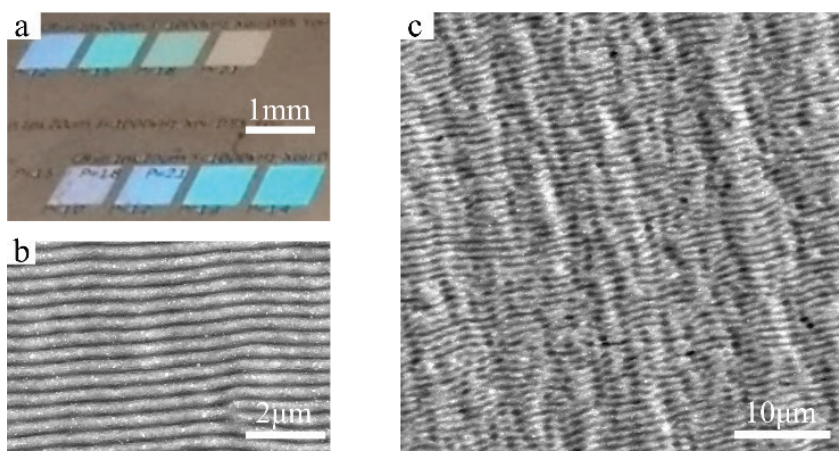

Fig. 5 LIPSS generated on copper leading to diffraction under crooked white light incidence. The spatial periodicity of the LIPSS is in the visible regime (b). As demonstrator structures after green multipulse ablation of aluminum show versatility (c).

generation of LIPSS after $515 \mathrm{~nm}$ irradiation on copper at a fluence of $0.8 \mathrm{Jcm}^{-2}$ in figure $5 \mathrm{~b}$ and on aluminum with $0.52 \mathrm{Jcm}^{-2}$ in figure $5 \mathrm{c}$. The polarization direction is horizontal and orthogonal LIPSS occur. To generate a colorful mark the LIPSS periodicity or direction can be changed, where a crooked white light incidence leads to colors like in figure 5a. The preferred method to tilt the LIPSS would be a motorized half wave plate to alter the angle of the electric field. The spatial periodicity changes only slightly with fluence [8]. Following, one valuable option would be to change the wavelength. However, highpower optical parametric oscillators for fast processing are not available so far.

\section{Laser path computation routines}

The core of this contribution concerns the transformation of a color pixel graphic into laser-scanning paths. To date there is a lack of CAM solutions for the coloration of specimen and this section elaborates the necessary steps. Starting with binary black/white and grey level marking the color separation and allocation is discussed. The implementation in Matlab is based on a CAM solution for complex $3 D$ workpieces [21] and open source available under the GPL [22].

\subsection{Import and pixel matrix transformation}

Figure 6 shows the principle workflow, where the original picture is imported, the resolution adjusted and available colormap attributed. Subsequently, this leads to the computed representation of the color laser marking.

After importing a pixel graphic, a physical dimension in terms of area per pixel must be set. Depending on the size of the picture and focal laser spot, a reduction of the resolution makes sense considering the limited visual perception. Solely skipping pixels does not lead to practical results. The human perception of a colored graphic is more complex and here the Floyd-Steinberg error diffusion algorithm used for dithering. This allows reducing the number of pixels, hence keeping a valid representation of the original graphic. Moreover, the same algorithm is used to generate black/white and grey scale versions of colored images by transformation with error diffusion.

Following the reduction and mapping of the pixel to real size, the attainable colors or levels of grey are imported into the system. Similar colors are mapped with a shortest

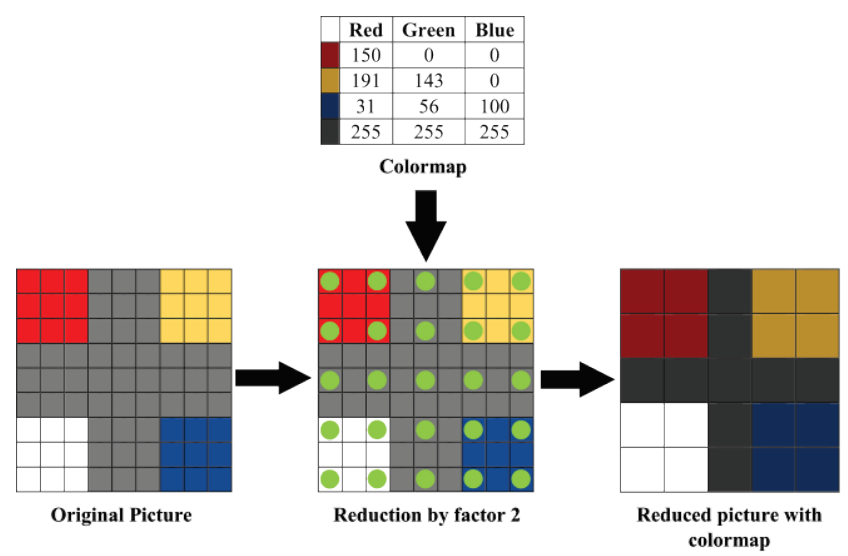

Fig. 6 Function of the laser path computation. The original picture is imported and reduced by dithering. A colormap of the attainable colors is imported and mapped.

distance approach in the red, green, and blue (RGB) color room to the color map from the material dependent parameter study. This leads to a reduced pixelated graphic with the attainable colors, as shown in figure 6.

\subsection{Strategies and machine code generation}

The reduced picture with the colormap or grey scale representation must be transformed into laser scan paths. To keep the process time low, same colors or grey levels are collected and laser machined line-wise. This reduces the duty time to settle the system after changing the average power. A skywrite feature is implemented to keep the irradiation condition and following energy dose controlled. Figure 7 depicts the list of parameters necessary for the computation. The spot size in $\mathrm{mm}$, feed rate of the optical

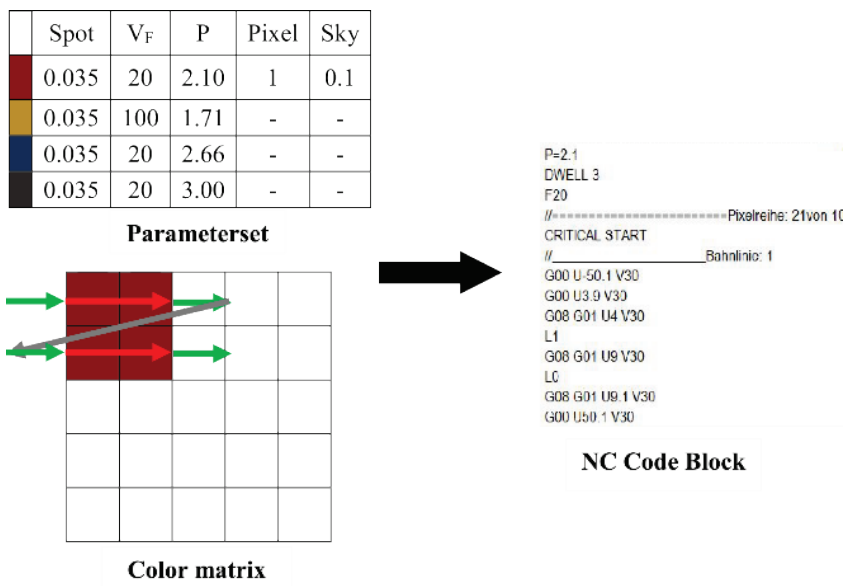

Fig. 7 Laser path computation with color matrices and generation of machine code. The green arrows depict the skywrite length and red the laser on segments. 
axes in $\mathrm{mm} / \mathrm{s}$ and laser power are defined for a certain color or grey level. Subsequently, the laser paths with the adjacent parameters are computed for line-wise modification with skywrite for each color matrix. If the pixels are smaller apart than a skywrite length, the scan speed is kept constant. Depending on the machine tool and axes configuration, the computed laser paths are translated into machine code. The export module is programmed flexible and following the routines can be widely used on different systems.

\section{Results and Discussion}

To demonstrate the capabilities of the software routines an experimental verification of the transform methods for greyscale color marking with pixel-to-path generation is presented.

\subsection{Greyscale marking of stainless steel}

An austenitic stainless-steel sample reveals the use of the dithering approach to generate high-contrast graphics. The colored high-resolution graphic is imported into the CAM software and two strategies used. A parameter study reveals the parameters to generate black marking and three grey levels. Table 1 gives an overview on the parameters used for this demonstrator. Figure 8 reveals the result of the introduced routines on the laser marking quality. Here, a comparison of greyscale and solely black marking is shown on a stainless $\mathrm{Cr} / \mathrm{Ni}$ steel 1.4301 . The four-level strategy clearly shows a more detailed representation of the original

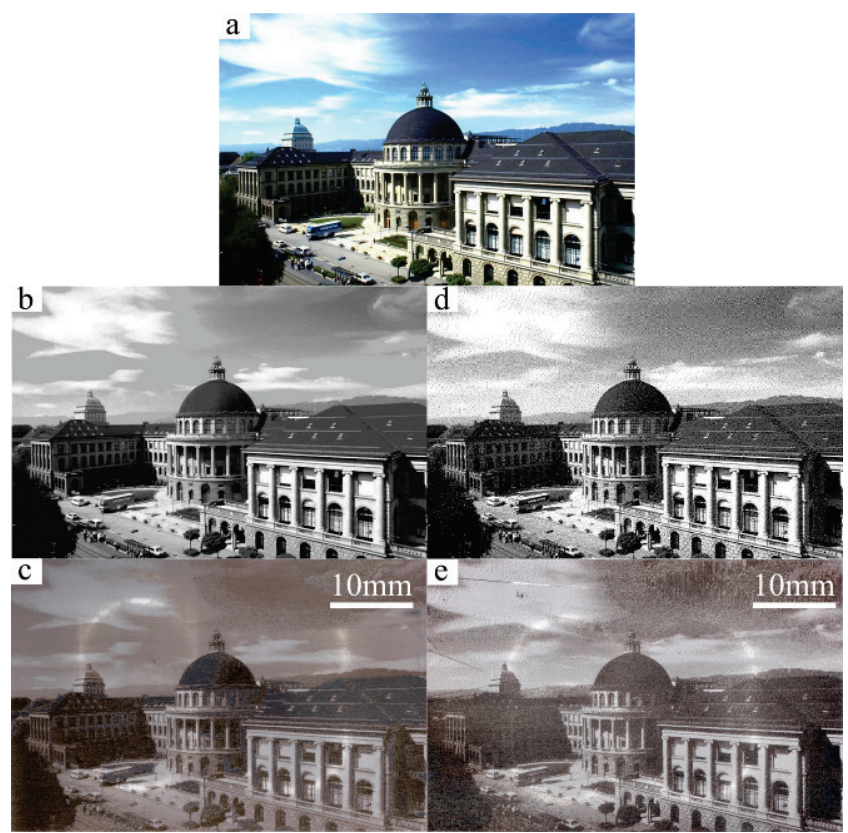

Fig. 8 Laser marking of a colored pixel image (a). Four grey level and dithering leads to the simulated graphic in (b) and the result on stainless steel in (c). Solely marking with black leads to different perception $(\mathrm{d}, \mathrm{e})$

picture. Especially, the details in the dark part of the building with two level window rows figure $8 \mathrm{c}$ are resolved better compared to solely black marking in figure $8 \mathrm{e}$. Moreover, the dithering and error diffusion is visible in the clouds above, where similar colored regions are packed into the same marking matrix. The total marking time depends on the pixel size and total geometrical expansion of the marking region. Here, the computation time was 7.3 min considering
Table 1 Processing parameters binary and grayscale marking on stainless steel. $515 \mathrm{~nm}$ wavelength with $10 \mathrm{ps}$ pulse duration, a laser spot of $30 \mu \mathrm{m}$, and $400 \mathrm{kHz}$ repetition rate.

\begin{tabular}{ccc}
\hline Color & Power $[\mathrm{W}]$ & Scanspeed $[\mathrm{mm} / \mathrm{s}]$ \\
\hline Binary & 11.6 & 500 \\
Grey 1 & 0.17 & 500 \\
Grey 2 & 2 & 500 \\
Grey 3 & 7.43 & 500 \\
Black & 16.6 & 500 \\
\hline
\end{tabular}

four grey levels, and 4.8 min for the binary black and white laser paths. The laser marking time was about one minute for both using a pixel size of $50 \mu \mathrm{m}$ and $10 \mu \mathrm{m}$ of line distance.

\subsection{Color marking of a titanium alloy}

A TiAl6V4 alloy serves as substrate for the coloration demonstrator. The attained color map of section 2.3 is used as a starting point and four induced colors selected for the proof of principle. Additionally, the titanium substrate surface allows a fifth color for high contrast, which is the untreated surface. The sample preparation of the surface influences the human perception of the marked image. Moreover, the laser parameters have to be adjusted for ground or polished surfaces, considering the change in absorptivity. Here, the titanium alloy was grinded up to grit 4000 with $\mathrm{SiC}$ paper and polished with $3 \mu \mathrm{m}$ alumina particles to mirror reflecting appearance. Adjacent laser parameters for blue, red, gold and black are listed in table 2.

Table 2 Processing parameters for coloration of titanium with $1030 \mathrm{~nm}$ wavelength, $10 \mathrm{ps}$ pulse duration, a laser spot of $35 \mu \mathrm{m}$, and $40 \mathrm{MHz}$ repetition rate.

\begin{tabular}{ccc}
\hline Color & Power $[\mathrm{W}]$ & Scanspeed $[\mathrm{mm} / \mathrm{s}]$ \\
\hline Blue & 6.5 & 60 \\
Red & 5.95 & 100 \\
Gold & 5.53 & 140 \\
Black & 10.3 & 20 \\
\hline
\end{tabular}

The original image in figure $9 \mathrm{a}$ has a resolution of $958 \times 717$ pixels and shows a sunset on the seaside. Similar to the grey-marking strategy, dithering is applied and a color matrix computed. All different colors are laser induced in subsequent line-like manner. The image is reduced one time by a factor of four and the computed result is shown in figure $9 \mathrm{~b}$ and magnified in 9d. The laser colored titanium surface reveals a conform mapping of the colors. Moreover, a magnification of the laser-machined region reveals the pixel size of $120 \mu \mathrm{m}$.

In order to push the process to the limit the original picture is manufactured with a pixel size of about $35 \mu \mathrm{m}$ and full resolution. Subsequently, the results in figure $9 \mathrm{f}-\mathrm{i}$ point to high-contrast coloration with a focal spot diameter as pixel size. Clearly, the magnified picture reveals the pixel size and no cross talk between the small laser-induced colors. This allows the coloration as small as the laser focal diameter leading to high resolution. 


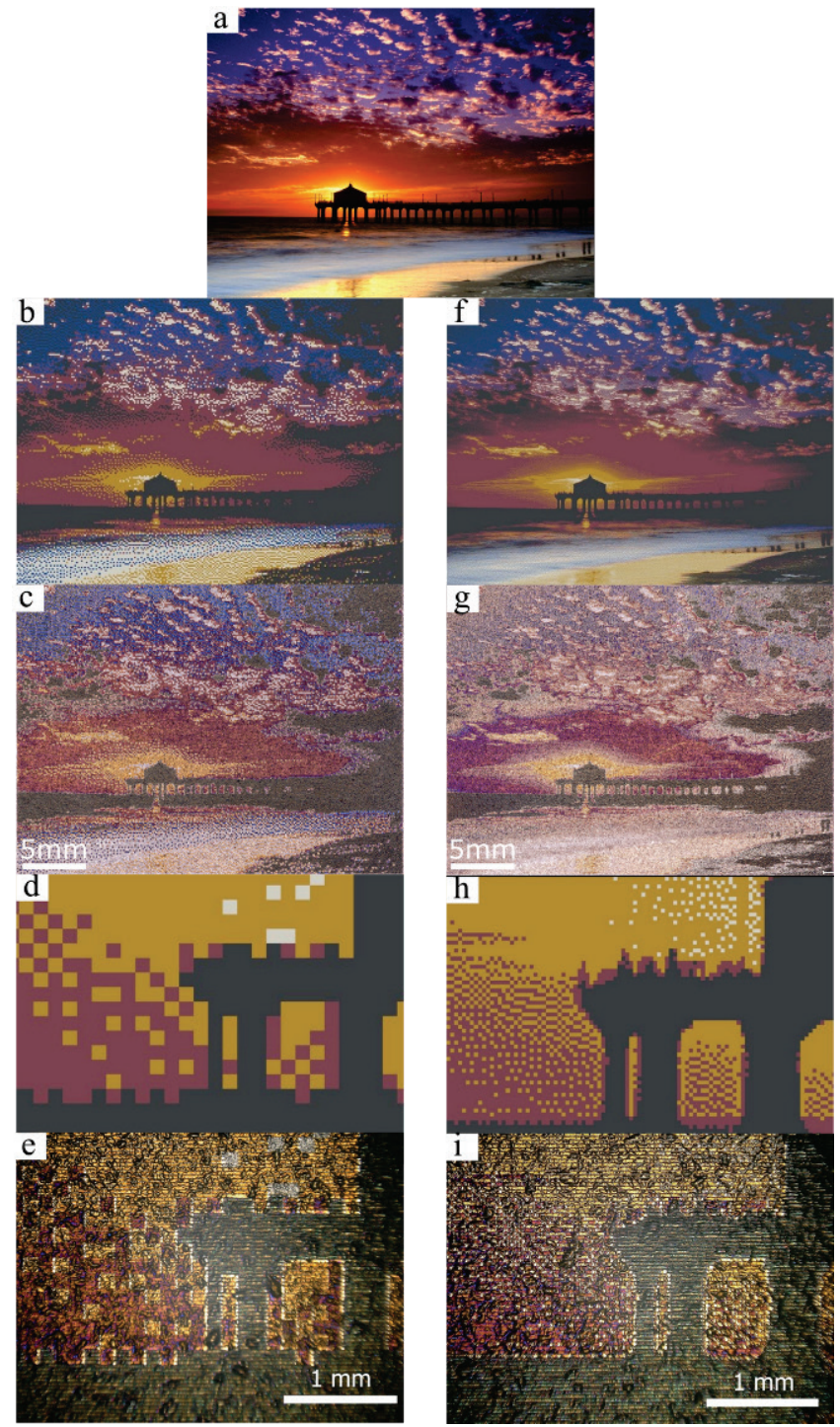

Fig. 9 Color marking of a sunset from the original picture (a), with fourfold reduced (left) and original (right) size. The simulated graphs $(b, d)$ show strong adherence to the laser colored result $(c, g)$. Magnification $(e, i)$ reveals a clear color separation and small pixel size

\section{Conclusions and outlook}

A routine for high-contrast laser marking and coloration was presented. The necessary computational approach routines has been discussed and points to a flexible marking utilizing strongly different physical processes. Moreover, the CAM software enables the transformation of a pixelated graphic into laser machining paths. The subroutines are flexible and, following, can be used on a number of experimental setups. A proof-of-concept on four level greyscale marking shows superior quality in comparison to binary black laser marking. Moreover, a demonstrator reveals vivid colors and show enables very good visual perception of a complex image on a titanium alloy.

The presented study concerning laser-produced diffraction gratings based on LIPSS will be expanded to laser mark complex graphics. Hence, the linear polarization will be tilted with a wave plate to alter the spatial directionality. This could be used for anti-counterfeiting color marking and the laser path calculation carried out with the presented software tool set. Additionally, an extended parameter assessment on the generation of resonant nanoparticles is planned. The experimental study on copper did not show any vivid color and oxidation processes of the nanoparticles could have played a role. Following, the laser processes will be carried out in vacuum and on noble metals.

\section{Acknowledgments}

The authors would like to thank the Swiss National Science Foundation [NSF $(\mathrm{CH})$ ] under the project "FuSSI Tools" (No. 169654) for financial support.

\section{References}

[1] A.J. Antończak, D. Kocoń, M. Nowak, P. Kozioł, and K.M. Abramski: Appl. Surf. Sci., 264, (2013) 229.

[2] Z.L. Li, H.Y. Zheng, K.M. Teh, Y.C. Liu, G.C. Lim, H.L. Seng, and N.L. Yakovlev: Appl. Surf. Sci., 256, (2009) 1582

[3] B. Dusser, Z. Sagan, H. Soder, N. Faure, J.P. Colombier, M. Jourlin, and E. Audouard: Opt. Express, 18, (2010) 2913.

[4] A.Y. Vorobyev, C. Guo: Appl. Phys. Lett., 92, (2008) 041914.

[5] J. Yao, C. Zhang, H. Liu, Q. Dai, L. Wu, S. Lan, A.V. Gopal, V.A. Trofimov, and T.M. Lysak: Appl. Surf. Sci., 258, (2012) 7625.

[6] T. Jwad, P. Penchev, V. Nasrollahi, and S. Dimov: Appl. Surf. Sci., 453, (2018) 449.

[7] J.-M. Guay, A. Calà Lesina, G. Côté, M. Charron, D. Poitras, L. Ramunno, P. Berini, and A. Weck: Nat. Commun., 8, (2017) 16095.

[8] N. Ackerl, P. Boerner, K. Wegener: J. Laser Appl., 31, (2019) 022501.

[9] H. Tao and J. Lin: Opt. Lasers Eng., 114, (2019) 31.

[10] F. Fraggelakis, G. Mincuzzi, J. Lopez, I. ManekHönninger, and R. Kling: Opt. Express, 25, (2017) 18131.

[11] C. Neugebauer, S. Quaranta, S. Ehrenmann, C. Rest, and J. Sadowitz: SPIE, Laser-Based MicroNanoprocessing XIII, (2019), 1090604.

[12] P. Laakso, H. Pantsar, H. Leinonen, and A. Helle: ICALEO 2008 - 27th Int. Congr. Appl. Lasers ElectroOptics, 405, (2008) 212.

[13] A.J. Antończak, Ł. Skowroński, M. Trzcinski, V. V. Kinzhybalo, Ł.K. Łazarek, and K.M. Abramski: Appl. Surf. Sci., 325, (2015) 217.

[14] E. Akman and E. Cerkezoglu: Opt. Lasers Eng., 84, (2016) 37

[15] V. Veiko, G. Odintsova, E. Vlasova, Y. Andreeva, A. Krivonosov, E. Ageev, and E. Gorbunova: Opt. Laser Technol., 93, (2017) 9.

[16] Y.M. Andreeva, V.C. Luong, D.S. Lutoshina, O.S. Medvedev, V.Y. Mikhailovskii, M.K. Moskvin, G. V. Odintsova, V. V. Romanov, N.N. Shchedrina, and V.P. Veiko: Opt. Mater. Express, 9, (2019) 1310.

[17] S. Tawfick, M. De Volder, D. Copic, S.J. Park, C.R. Oliver, E.S. Polsen, M.J. Roberts, and A.J. Hart: Adv. Mater., 24, (2012) 1628.

[18] E.I. Ageev, V.P. Veiko, E.A. Vlasova, Y.Y. Karlagina, A. Krivonosov, M.K. Moskvin, G. V. Odintsova, V.E. Pshenichnov, V. V. Romanov, and R.M. Yatsuk: Opt. Express, 26, (2018) 2117. 
[19] R.L. Higginson, C.P. Jackson, E.L. Murrell, P.A.Z. Exworthy, R.J. Mortimer, D.R. Worrall, and G.D. Wilcox: Mater. High Temp., 32, (2015) 113.

[20] J. Bonse, S. Hohm, S. V Kirner, A. Rosenfeld, and J. Kruger: IEEE J. Sel. Top. Quantum Electron., 23, (2017) 9000615.

[21] N. Ackerl, M. Warhanek, J. Gysel, and K. Wegener: Int. J. Adv. Manuf. Technol., 103, (2019) 1105.

[22] P. Gugger: GitHub Rep., (2019) http://doi.org/ $10.5281 /$ zenodo. 2711921 .

(Received: May 20, 2019, Accepted: January 15, 2020) 